\title{
PHYSICAL MODELING OF METAMATEREALS ON THE BASIS OF THE FLAT SPIRAL COIL
}

\author{
Z.R. Idiatullov, I.I. Ahmetov, M.M. Fatyhov, S.V. Spiridonov, I.M. Lerner \\ Kazan National Research Technical University named after A. N. Tupolev - KAI, Kazan, Russia; \\ German-Russian Institute of Advanced Technologies (GRIAT)
}

DOI: $10.36724 / 2664-066 X-2020-6-4-\mid 8-30$

\begin{abstract}
In this work, the problems of physical modeling of metamaterials based on flat spiral coils with different geometric shapes were considered. The main parameters of the structures metamaterial modeling, methods of creation, as well as their unique properties are considered. As a result of physical modeling, structures were obtained that model the metamaterial with different values of dimensions and parameters. On the basis of the experimental data, the best variant of the geometric shape of the structure element was determined, based on the highest transmission coefficient.
\end{abstract}

KEYWORDS: metamaterial, electromagnetic interaction, physical modelling.

\section{INTRODUCTION}

In recent years, metamaterials have attracted great attention in connection with the prospects of their practical application for radio masking and the development of antenna technology - the creation of compact flat antennas, high-impedance surfaces, decoupling of antenna array elements. Metamaterials are artificial materials consisting of composite structural elements that acquire their unusual properties that do not exist in nature. A fundamental feature of metamaterials is their ability to provide a strong magnetic response at terahertz and even optical frequencies, where the magnetic permeability of ordinary materials is close to unity.

In accordance with various physical properties, metamaterials can be classified into acoustic, thermal, photonic and electromagnetic (EM) metamaterials. Recently, EM metamaterials that are constructed from subwavelength structural units have received much attention because of their exotic properties, such as the inverse Doppler effect, Vavilov-Cherenkov back radiation, negative refractive index, double negative property, etc. EM-metamaterials simultaneously have negative permittivity and negative permittivity (ie, e $<0$ and $\mu<0$ ) and obey the left-hand rule, which differ from conventional materials.

Currently, the transmission efficiency is strictly limited in a wireless power transmission system (WPT) with conventional materials and therefore new methods are required to increase the transmission efficiency. This problem can be solved with the help of metamaterials, which are special structures. Flat spiral coils can be used as elements for physical modeling, and for predicting the behavior of electromagnetic fields in metamaterials. The relevance of this topic is justified by the fact that today metamaterials have unique properties with which you can increase the efficiency of energy transfer. It is also important to note that it allows you to save money on the transmission and reception of electrical energy, as well as reduce the costs associated with maintaining direct connectors. 


\section{THEORETICAL PART}

A. Areas of application of metamaterials and their basic properties

According to different values of permittivity and permeability, materials can be divided into four categories (Figure 1).

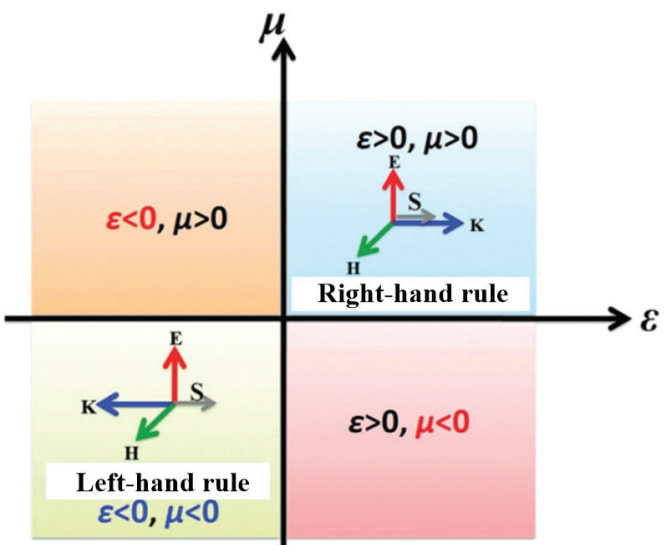

Fig. 1. Categories of materials based on values of different dielectric constant (e) and permeability (m).

E, H, K and S - electric field, magnetic field, wave propagation and Poynting vectors

For ordinary materials, dielectric constant and permeability are positive. When the dielectric constant or permittivity is negative (i.e., $\mathrm{e}<0$ and $\mu>0$ or e $>0$ and $\mu<0$ ), the materials are called epsilon-negative (e.g. metals, plasma) or $\mu$-negative materials (e.g. gyrotropic magnetic materials). Interestingly, when these two parameters are simultaneously negative, the materials are defined as double negative materials. In fact, a double negative property was originally observed in metamaterials.

When the values of $\mathrm{e}$ and $\mathrm{m}$ are simultaneously positive or negative, electromagnetic waves can propagate through the medium. For ordinary materials (i.e., e $>0$ and $\mu>0$ ), the electric vector $E$, the magnetic vector $\mathrm{H}$ and the wave vector $\mathrm{K}$ correspond to the righthand rule. Meanwhile, the Poynting vector $\mathrm{S}$, which is the energy flux density, has the same direction of wave propagation when the energy decays along with wave propagation.

For metamaterials with a double negative property (e $<0$ and $\mu<0$ ), electromagnetic waves can propagate through the media and satisfy the Maxwell equations, equation (1) - (4),

$$
\begin{aligned}
& \mathrm{k} \times \mathrm{E}=\omega \mu \mathrm{H} \\
& \mathrm{k} \times \mathrm{E}=-\omega \mu \mathrm{H} \\
& \mathrm{k} * \mathrm{E}=0 \\
& \mathrm{k} * \mathrm{H}=0
\end{aligned}
$$

When the permittivity and permittivity are both negative, the three vectors $\mathrm{E}, \mathrm{H}$, and $\mathrm{K}$ of the double negative materials obey the left-side rule, and the Poynting vector is opposite to the direction of the wave vector that is directed to the source. In this case, it is shown that the energy flux of electromagnetic waves is opposite to the positive direction of the source phase.

The simplest example of the difference between normal materials and a negative refractive index (NRI) is obtained by comparing two materials for the refractive effect. In a normal transparent (dielectric) material, an electromagnetic beam is refracted in the normal direction (blue line) when it passes from air to a material with a higher optical density (positive index), as shown in the figure. In NRI, the beam is refracted to the other side (red line) of the normal, as shown in Figure 2.

The angle of refraction is obtained from Snell's law, which states that the ratio of the sines of the angles of incidence and refraction is equal to the ratio of the velocities in two media or, equivalently, the inverse ratio of the refractive indices $n_{1}$ and $n_{2}$. Thus,

$$
n_{1} \sin \theta_{1}=n_{2} \sin \theta_{2}
$$

where $n_{1}, n_{2}, \theta_{1}$ and $\theta_{2}$ - are the refractive index of media 1 and 2 , the incident and refractive angles.

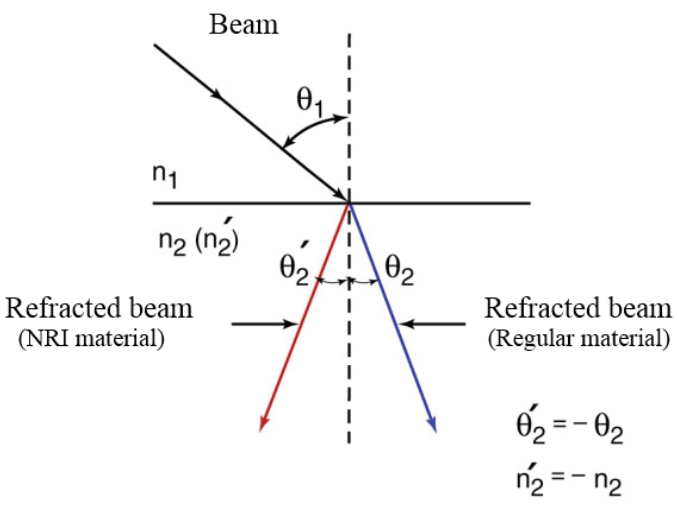

Fig. 2. Refraction in normal material (blue line) and NRI (red line)

Figure 2 shows the practical application of this material with NRI. In this figure, we compare the effects of focusing the light of lenses made from ordinary materials (blue) and NRI (red). Note that NRI has an effect opposite to that of ordinary material: in a convex lens, rays diverge, and in a concave lens, rays converge. In Figure 3, a plate containing normal material (a) deflects the beam $\left(s_{1}\right)$ so that a virtual source is visible inside the material, and a plate containing NRI (b) focuses the beam $\left(s_{1}\right)$ inside the material $\left(f_{1}\right)$ and on the other side of source outside the material $\left(f_{2}\right)$ [1].

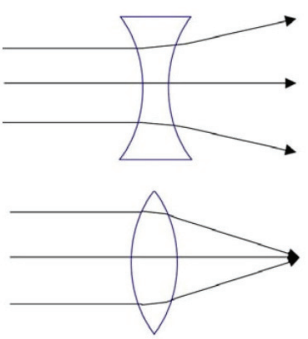

(a)
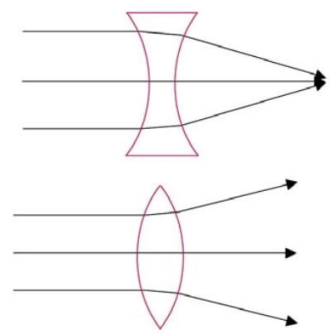

(b)
Fig. 3. The effect on the bundles of EM lenses made from (a) normal material and (b) NRI 


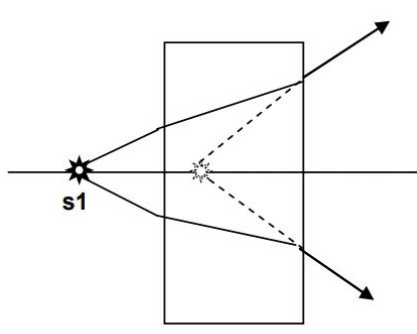

(a)

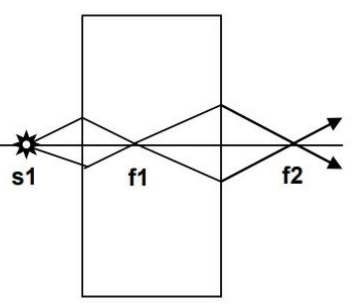

(b)
Fig. 4. The effect on electromagnetic beams of flat plates made of (a) normal material and (b) NRI

For metamaterials with negative permittivity and negative permittivity, the incident beam and the refracting beam are on the same side of the normal. This indicates that the refractive index of metamaterials is a negative value, namely $n=-\sqrt{e_{\mu}}$.

In addition, metamaterials have other unique properties, including the inverse Doppler effect, Vavilov - Cherenkov back radiation.

The Doppler effect (discovered in 1842 by Christian Doppler) is that with the relative movement of the observer and the wave source, the wave frequency changes. If we are talking about a sound wave, for example, from an approaching train, then its frequency increases as it approaches, when the train moves away, the frequency decreases. The same phenomenon occurs with light. When the object and the observer approach each other, the frequency of the light wave increases (from red to blue), and, conversely, if they move away, the frequency decreases.

To observe this effect, it is necessary to grow a nanostructured silicon crystal, a unique photonic superprism with a negative refractive index. By directing the laser beam to the prism and changing the distance between it and the detector, the scientists managed to register the opposite Doppler effect, when the light "turns red" when objects are approaching, and "turn blue" when removed [2].

We note that the Vavilov - Cherenkov radiation (IVF) of the charge in the "left" medium is "inverse" in nature, that is, it propagates (in the sense of the direction of the energy flux density) at an obtuse angle to the velocity of the charge. In the case when the charge flies into the "left" medium, the ion-frequency generator generated in it falls to the interface, generating radiation, which can be called "reverse Cherenkov-transitional radiation" (RCTR). Radiation exists only in the presence of an interface (as transition radiation) and only in the presence of an inverse IVF in a medium [3].

Metamaterials can be created with various electrical properties. Their classification depending on the values of the relative dielectric and relative magnetic permeabilities is shown in Figure 5.

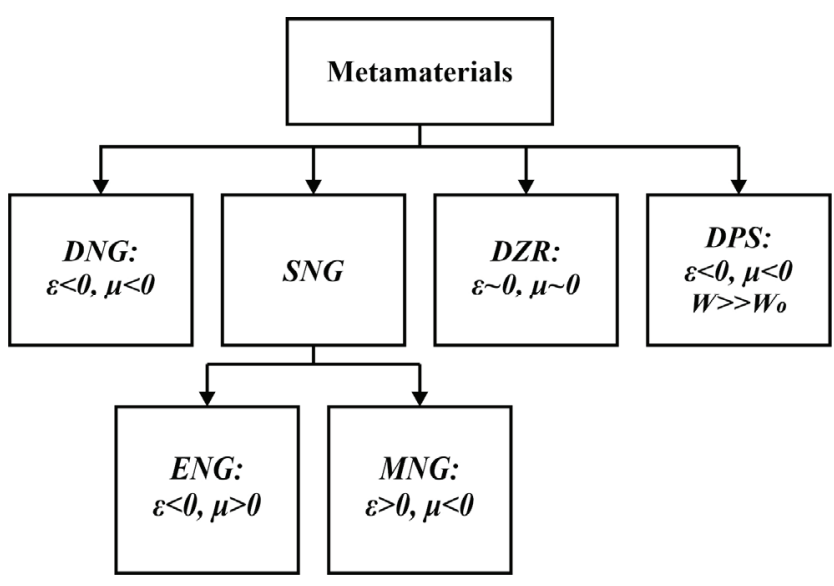

Fig. 5. Classification of metamaterials

An analysis of this figure shows that the names MM determine the relative permeabilities of the material:

- DNG - double negative (both permeabilities are negative);

- DPS - double positive (both permeabilities are positive, the wave resistance of the medium $\mathrm{W}$ is much higher than the wave resistance of the free space $\mathrm{W}_{0}$. Hence the second name - - Hi-Z - high impedance surfaces);

- NG - single negative (one value from the permeabilities is negative: if the relative dielectric constant is negative, then the name ENG is accepted, if the relative magnetic permeability, then MNG). These materials are also called mixed-type metamaterials;

- DZR - double zero (both permeabilities are practically equal to zero, which leads to the fact that the reflection coefficient from these materials is close to zero).

The electrical properties of metamaterials can either vary with frequency, i.e. form dispersing media, or keep their values in a relatively wide frequency band. In the first case, MMs form frequency-selective structures (FSS), as shown in Figure 6.

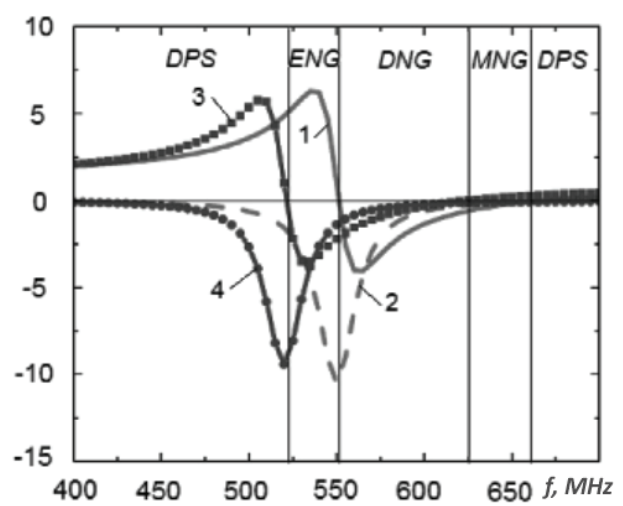

Fig. 6. Frequency dependence of dispersive type metamaterials: $1-\varepsilon_{r}^{\prime} ; 2-\varepsilon_{r}^{\prime \prime} ; 3-\mu_{r}^{\prime} ; 4-\mu_{r}^{\prime \prime}$ 
The main feature of the construction of metamaterials is the artificially created periodic structure. Depending on the number of directions the frequency is observed, $1 \mathrm{D}$, $2 \mathrm{D}$ and $3 \mathrm{D}$ structures are distinguished. The physical implementation of FSS may be different. So, they can be implemented in the form of dielectric materials with fillings in the form of open resonant wire rings (SRR).

The shape of the rings can be deformed from round to square. Examples of designs are shown in Figure 7 a, b. For the physical implementation of metamaterials that retain their electrical properties in a wide frequency band, rings are not closed, but closed. In addition, the rings can be arranged randomly in the material structure. Two options for implementing this design from the work are shown in Figure 8 [4].

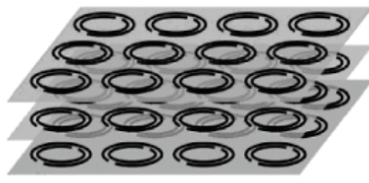

(a)

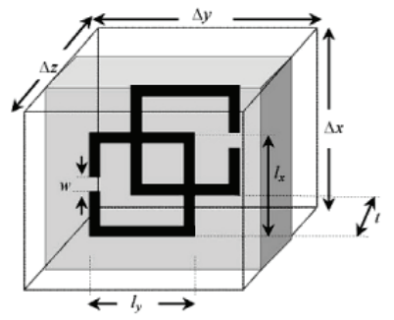

(b)
Fig. 7. Examples of the physical implementation of frequencyselective structures: a - with filling from open rings; $\mathrm{b}$ - with filling from open squares

In accordance with their specific electromagnetic properties, metamaterials have great potential applications in the development of invisible cloaks, super lenses, absorption of electromagnetic waves and microstrip antennas. In recent years, it is known that negative-permeability metamaterials can improve the resolution of magnetic resonance imaging, due to their ability to focus radio frequency magnetic field lines.

In addition, the unique properties of metamaterials, especially the amplification of damped waves, are of great importance for WPT, since resonant coupling is, in fact, a coupling of damped waves. When the metamaterial is placed between the coils of the transmitter and receiver, the damped wave can be amplified, and the transmission coefficient can also improve, which leads to an increase in the energy transfer efficiency and transmission distance.

\section{B. Principles of operation of metamaterials}

We begin the explanation of the metamaterials working principles with the simplest version of their execution - high-impedance structures. These designs are resonant $\mathrm{LC}$ circuits tuned to a specific frequency. The elements of these circuits, as shown in Figure 9, are formed as follows: capacitances are created in the gaps between the metal plates of one row or adjacent rows, and the inductance is formed due to the vortex-like leakage of the magnetic field into the internal cavity. High-impedance surfaces are used as shields for controlling the electromagnetic field located near wire antennas.

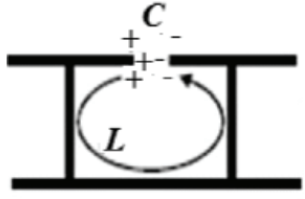

Fig. 8. Representation of a Hi-Z cell as an LC circuit

In the classical construction of an antenna with a metal reflector in the form of a plane (PEC), the emitter is placed at a distance of $\lambda / 4$ ( $\lambda$ is the working wavelength) from the metal screen, as shown in Figure 9 a. In this case, the electr agnetic wave incident on the screen from the emitter and reflected from the screen in the region located behind the emitter is added in antiphase, and the diffraction maximum occurs only in the direction of the main lobe of the radiation pattern. When the emitter is placed at a distance less than $\lambda / 4$, as shown in Figure 9b, these waves are not in antiphase and a diffraction lobe appears, comparable in level with the main lobe of the antenna pattern.

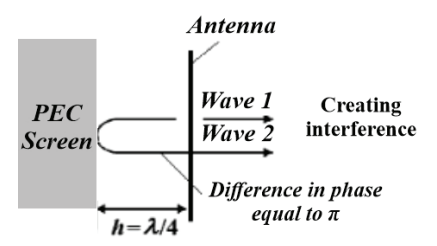

(a)

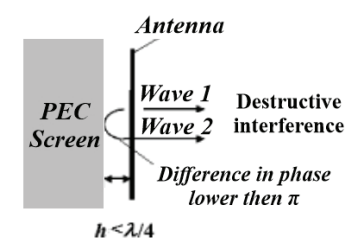

(b)
Fig. 10. Placing the emitter near a perfectly conducting electric current screen: a - case of suppression of the diffraction lobe; $\mathrm{b}$ - the case of the formation of a diffraction lobe

Near the high-impedance surface, the phase shift between the incident and reflected waves is zero. As a result, when the emitter is placed at distances much shorter than the wavelength, the diffraction lobe does not occur, as shown in Figure 10. This effect is due to the fact that a resonant cavity is formed between the metal base and the emitter, acting as a partially reflecting surface [4].

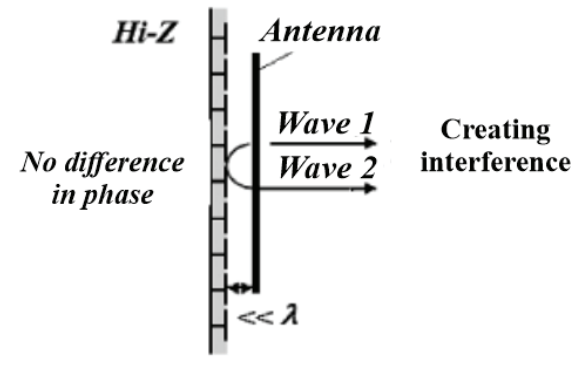

Fig. 11. Placing the emitter near Hi-Z

\section{Magnetic field of a flat spiral coil}

The magnetic field of a conductor with a current of arbitrary configuration (for example, a coil) can be obtained by the known method of superposition of the fields of elementary linear sections of the conductor. 


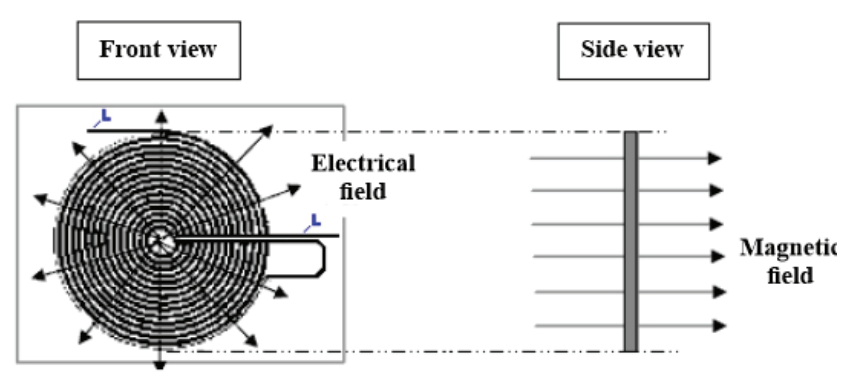

Electromagnetic coil

Fig. 12. The structure of the magnetic field lines in the area adjacent to the turns of a flat spiral coil

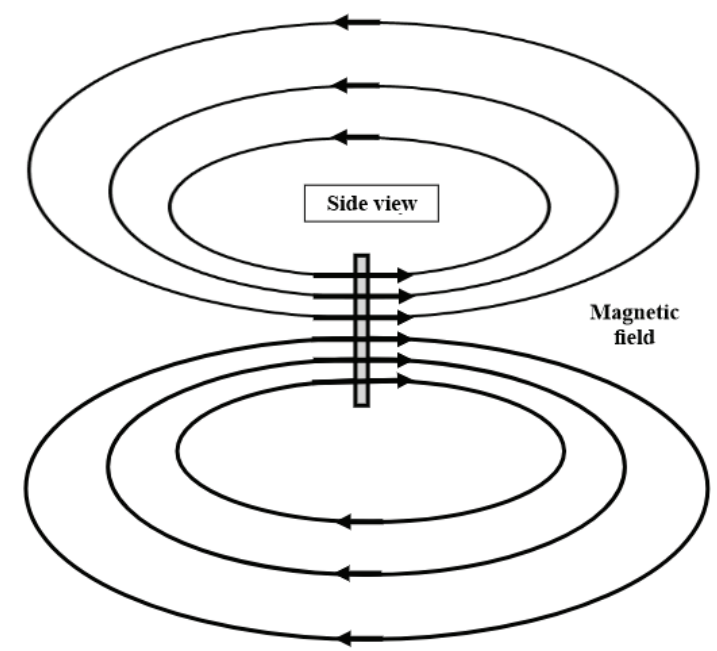

Fig. 13. The generally accepted structure of the magnetic field lines in the near zone (coil zone) of a flat spiral coil outside

The magnetic field of a flat spiral coil can be represented by a superposition of the fields of concentric individual turns, the diameter of which varies in accordance with the pitch of the spiral. The turn consists of infinitely small individual straight-line sections. In this case, the magnetic field of each turn is calculated as a superposition of the fields of its constituent discrete sections (DE). The numerical solution of the mathematical model constructed in this way allows us to obtain the value of the magnetic field induction of a complex configuration of conductors at any point in space.

The implementation of this model for a flat coil includes the following steps:

1. Calculation of the magnetic induction of the DE field at any point in space (PiS) according to the Bio-Savard-Laplace law:

$$
\Delta \mathbf{B}(\mathbf{r})=\frac{\mu_{0} I}{4 \pi}\left(\frac{\Delta \mathbf{L} \times \mathbf{r}}{\mathbf{r}^{3}}\right)
$$

where $\quad \Delta \mathbf{L}=\left(\Delta L_{x} ; \Delta L_{y} ; \Delta L_{z}\right) \quad-\quad$ discrete section; $\mathbf{r}=\left(r_{x} ; r_{y} ; r_{z}\right)-$ research point.

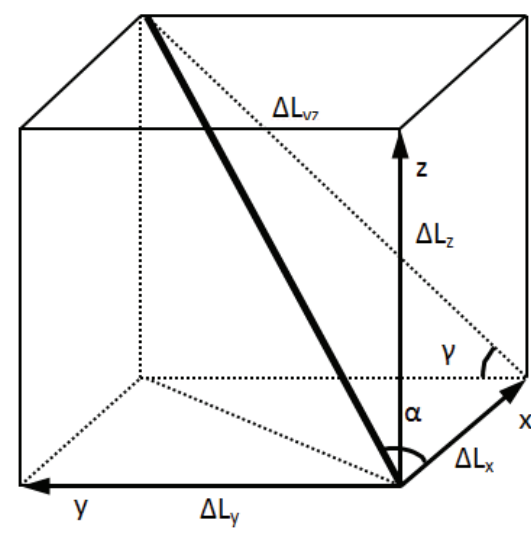

Fig. 14. Determination of orientation DS

If the vector product $\Delta \mathbf{L} \times \mathbf{r}$ is denoted by the auxiliary vector $\mathbf{k}(\mathbf{k}=\Delta \mathbf{L} \times \mathbf{r})$, then, taking into account formulas (7), it follows (8) and (9):

$$
\begin{aligned}
& \Delta L x=\Delta L \cos \alpha ; \Delta L_{y}=\Delta L \sin \alpha \cos \gamma ; \\
& \Delta L_{z}=\Delta L \sin \alpha \sin \gamma \\
& |\mathbf{k}|=|\Delta \mathbf{L} \times \mathbf{r}|=\sqrt{k_{x}^{2}+k_{y}^{2}+k_{z}^{2}} ; \\
& \mathrm{k}_{x}=\left|\begin{array}{cc}
\Delta L_{y} & \Delta L_{z} \\
r_{y} & r_{z}
\end{array}\right|=\Delta L_{y} r_{z}-r_{y} \Delta L_{z} ; \\
& \mathrm{k}_{y}=\left|\begin{array}{cc}
\Delta L_{z} & \Delta L_{x} \\
r_{z} & r_{x}
\end{array}\right|=\Delta L_{z} r_{x}-r_{z} \Delta L_{x} \text {; } \\
& \mathrm{k}_{z}=\left|\begin{array}{cc}
\Delta L_{x} & \Delta L_{y} \\
r_{x} & r_{y}
\end{array}\right|=\Delta L_{x} r_{y}-r_{x} \Delta L_{y} \text {; } \\
& |\mathbf{r}|=\sqrt{\mathrm{r}_{\mathrm{x}}^{2}+\mathrm{r}_{\mathrm{y}}^{2}+\mathrm{r}_{\mathrm{z}}^{2}} \\
& r_{x}=x_{i}-x ; r_{y}=y_{i}-y ; r_{z}=z_{i}-z ;
\end{aligned}
$$

where: $(x i, y i, z i)$ are the coordinates of the remote control; $(x, y, z)$ - coordinates of an arbitrary point in space.

2. Calculation of the magnetic field of a coil:

For this, a consistent calculation of the influence of all sites is necessary, taking into account their spatial location and the linear dimensions of the projections of the sites.

To simplify the calculation, the coil is placed in one of the planes of space ( $x y$ plane) (see Figure 13). Then the projections of the remote control are defined as:

$$
\begin{array}{ll}
\Delta L_{x i} & =\Delta L \cdot \sin \alpha ; x_{i}=R \cdot \cos \beta ; \\
\Delta L_{y i} & =\Delta L \cdot \cos \alpha ; y_{i}=R \cdot \sin \beta ; \\
\Delta L_{z i} & =0 ; \quad z_{i}=0 .
\end{array}
$$


where: $\mathrm{i}=1 \ldots \mathrm{N} ; \beta=0 \ldots 360^{\circ}$ in increments of $2 \pi / \mathrm{N}$; $\Delta \mathrm{L}=2 \pi \mathrm{R} / \mathrm{N} ; N-$ the number of sections of the coil (determines the accuracy of the calculations); $R$ - turn radius.

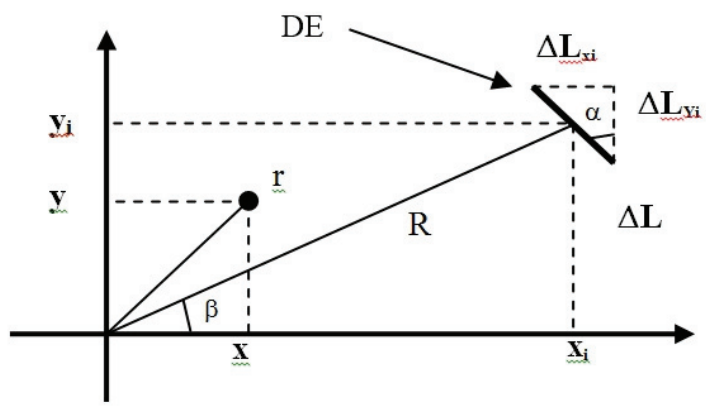

Fig. 14. Straight line discrete section (DS)

3. Calculation of the total magnetic induction in PiS:

It is carried out by the principle of a superposition of induction of turns creating a spiral:

$$
\mathrm{B}_{\text {full }}=\mathrm{B}_{\mathrm{R} 1}+\mathrm{B}_{\mathrm{R} 2}+\ldots+\mathrm{B}_{\mathrm{Rm}-1}+\mathrm{B}_{\mathrm{Rm}}
$$

where $R_{m}$ is the radius of the outer coil (ring) of the spiral.

This simulation by measurement allows you to visualize the structure of the magnetic field using the well-known classical method.

\section{RESEARCH METHODS OF PRODUCING METAMATERIALS}

The frequency range of metamaterials extends to the terahertz, infrared and visible frequency ranges. The size of the structure for metamaterials depends on the practical application, as well as on the methods of synthesis and manufacture. In the field of microwave frequencies, metamaterials are mainly manufactured by lithography with a shadow mask; in the terahertz or visible frequency range, metamaterials can be obtained by electron or ion beam lithography.

Figure 15 shows metamaterials with various sizes and structures. The first manufacturing method is the nanofabrication method to obtain metamaterials consisting of nanoscale U-shaped unit cells of gold in Figure 15a. Compared to previous double ring resonators, this single gold ring matrix can generate magnetic resonance and achieve a negative refractive index of about $100 \mathrm{THz}$, which is the result of the resonance of the inductive-capacitor circuit.

The second method is electron beam lithography for the manufacture of multilayer $\mathrm{Ag}-\mathrm{MgF} 2-\mathrm{Ag}$ structural metamaterials that were located on a glass substrate coated with a thin film of $5 \mathrm{~nm}$ thick indium tin oxide to avoid charging effects during lithography. As shown in Figure 15b, the structural unit was a nanoscale grid, and a negative refractive index was observed at a wavelength of almost $780 \mathrm{~nm}$ in silver-based metamaterials.
The copper-based metamaterial was prepared by lithography using a shadow mask, and the microdimensional structure nodes were composed of split-cavity ring resonators (SRR) and copper wires in Figure $15 \mathrm{~g}$, which showed negative permittivity and negative permeability in the microwave mode.

Figure 15 e shows the method of surface multilayer galvanic synthesis of non-planar copper-based SRRs on a flexible silicon substrate.
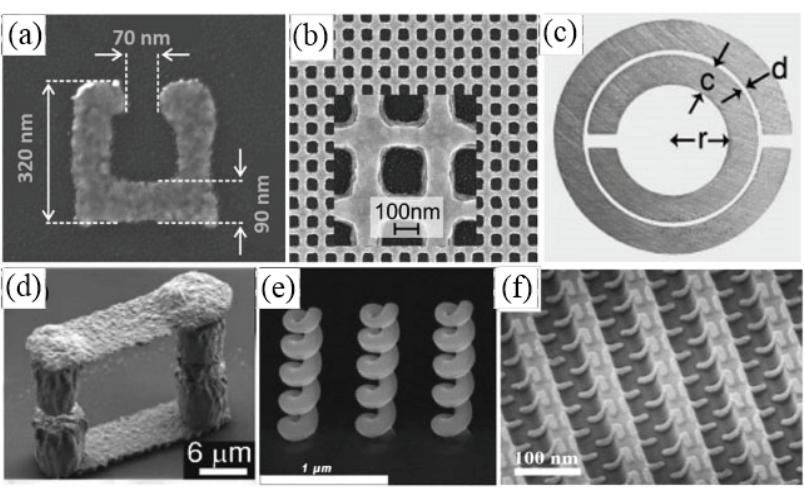

Fig. 15. Metamaterials with various sizes and structures. (a-f) Metamaterials with a nanoscale U-shaped, nanoscale mesh, round, flexible metamaterial with a micronized U-shaped structure, nanoscale spiral and nanoscale L-shaped [1]

\section{EXPERIMENTAL PART}

The figures show various geometric forms of structures simulating a metamaterial. In this section, the parameters of the coils and their calculations will be presented.

\section{Flat disk inductance (coils)}

The flat disk inductance (coil) is found by:

$$
\mathrm{L}=\frac{\mu_{0}}{8 \pi} \omega^{2} \mathrm{~d} \pi
$$

where $\omega$ is the number of turns of the coil; $d=(d 1+d 2) / 2$ - its average diameter; $\Psi$ is the value whose values are given in table 1 depending on the ratio $\rho=r / d$, where $r$ is the radial size (width of the winding) of the coil and the magnetic constant $\mu 0=4 n^{*} 10^{-7} \mathrm{GN} / \mathrm{m}$.

For small values of $\rho$, the interpolation according to Table 1 may turn out to be insufficiently accurate, and then to determine $\Psi$ one should use the formula:

$$
\begin{aligned}
& \pi=4 \pi\left[\left(1+\frac{\mathrm{p}^{2}}{24}+\frac{11}{2880} \mathrm{p}^{4}+\ldots\right) \ln \frac{4}{\mathrm{p}}\right. \\
& \left.-\frac{1}{2}+\frac{43}{288} \mathrm{p}^{2}+\frac{1}{150} \mathrm{p}^{4}+\ldots\right] .
\end{aligned}
$$

On the contrary, with large $\rho(\rho>0,5)$ :

$$
\begin{aligned}
& \pi=\frac{(1+p)^{3}}{p^{2}}\left(1,7424+3,2900{ }^{3} \ln \pi-2,27 \pi^{3}+0,3702\right. \\
& \left.\pi^{5}+0,0826 \pi^{7}+0,0312 \pi^{9}+\ldots\right),
\end{aligned}
$$


Table 1

Values $\Psi$ for flat reel

\begin{tabular}{r|r||r|r||r|r||r|c}
\hline$\rho$ & $\Psi$ & $\rho$ & $\Psi$ & $\rho$ & $\Psi$ & $\rho$ & $\Psi$ \\
\hline 0,00 & & & & & \\
\hline 01 & 69,008 & 26 & 28,767 & 0,50 & 20,601 & 0,75 & 16,360 \\
02 & 60,299 & 27 & 27,290 & 51 & 20,381 & 76 & 16,235 \\
03 & 55,206 & 28 & 27,392 & 52 & 20,165 & 77 & 16,112 \\
04 & 51,595 & 29 & 26,968 & 53 & 19,955 & 78 & 15,992 \\
0,05 & 48,794 & 0,30 & 26,560 & 0,55 & 19,750 & 79 & 15,874 \\
06 & 46,507 & 31 & 26,166 & 56 & 19,354 & 0,80 & 15,759 \\
07 & 44,574 & 32 & 25,786 & 57 & 19,162 & 82 & 15,646 \\
08 & 42,902 & 33 & 25,418 & 58 & 18,976 & 83 & 15,536 \\
09 & 41,428 & 34 & 25,063 & 59 & 18,793 & 84 & 15,323 \\
0,10 & 40,111 & 0,35 & 24,719 & 0,60 & 18,614 & 0,85 & 15,220 \\
11 & 38,920 & 36 & 24,386 & 61 & 18,440 & 86 & 15,119 \\
12 & 37,835 & 37 & 24,063 & 62 & 18,269 & 87 & 15,021 \\
13 & 36,838 & 38 & 23,750 & 63 & 18,102 & 88 & 14,925 \\
14 & 35,916 & 39 & 23,446 & 64 & 17,939 & 89 & 14,832 \\
0,15 & 35,058 & 0,40 & 23,150 & 0,65 & 17,779 & 0,90 & 14,740 \\
16 & 34,258 & 41 & 22,863 & 66 & 17,623 & 91 & 14,650 \\
17 & 33,507 & 42 & 22,584 & 67 & 17,470 & 92 & 14,563 \\
18 & 32,800 & 43 & 22,313 & 68 & 17,320 & 93 & 14,478 \\
19 & 32,132 & 44 & 22,049 & 69 & 17,174 & 94 & 14,394 \\
0,20 & 31,500 & 0,45 & 21,792 & 0,70 & 17,032 & 0,95 & 14,314 \\
21 & 30,900 & 46 & 21,541 & 71 & 16,891 & 96 & 14,235 \\
22 & 30,330 & 47 & 21,297 & 72 & 16,754 & 97 & 14,158 \\
23 & 29,785 & 48 & 21,059 & 73 & 16,620 & 98 & 14,083 \\
24 & 29,265 & 49 & 20,827 & 74 & 16,489 & 99 & 14,010 \\
0,25 & 28,767 & 0,50 & 20,601 & 0,75 & 16,360 & 1,00 & 13,939 \\
& & & & & & & \\
& & & & &
\end{tabular}

\section{E. Inductance of flat polygonal coils}

The average diameter $d$ of the equivalent planar circular coil is found from the diameter $D$ of the circle described around the middle coil of the coil. Having found the average diameter $\mathrm{d}$ of the coil, we determine its inductance $L$.

If the radial size $r$ is small compared with the diameter $D$, then the inductance of the coil can be determined directly by the following formulas:

For $\mathrm{n}=3$

$\mathrm{L}=\frac{3}{2 \pi} \mu_{0} \omega^{2} \mathrm{c}\left(\ln \frac{1}{p}+0,0945+0,4132 \mathrm{p}+0,3194 \mathrm{p}^{2}+\ldots\right)$;

For $\mathrm{n}=4$

$\mathrm{L}=\frac{2}{\pi} \mu_{0} \omega^{2} \mathrm{c}\left(\ln \frac{1}{p}+0,7260+0,1776 \mathrm{p}+0,125 \mathrm{p}^{2}+\ldots\right) ;$

$\mathrm{n}=6$

$\mathrm{L}=\frac{3}{\pi} \mu_{0} \omega^{2} \mathrm{c}\left(\ln \frac{1}{p}+1,3485+0,0678 \mathrm{p}+0,0491 \mathrm{p}^{2}+\ldots\right) ;$

For $\mathrm{n}=8$

$\mathrm{L}=\frac{4}{\pi} \mu_{0} \omega^{2} \mathrm{c}\left(\ln \frac{1}{p}+1,7120+0,0363 \mathrm{p}+0,0277 \mathrm{p}^{2}+\ldots\right)$.

F. Calculation of the inductance of structures simulating a metamaterial:

1) Square shape flat coil:

$\mathrm{c}=8,5 \mathrm{~cm} ; \mathrm{r}=3,5 \mathrm{~cm} ; \omega=5 ; \mathrm{D}=\frac{c}{\sin \frac{\pi}{n}}, \mathrm{~cm} ; \mathrm{D}=12 \mathrm{~cm}$; $\frac{r}{D}=\frac{3,5}{12}=0,3 ; \mathrm{d}=0,8075^{*} 12=10 \mathrm{~cm} ; \rho=\frac{3,5}{8,5}=0,4 ;$

$\ln \frac{1}{0,4}=0,9 ; 0,1776 * \mathrm{p}=0,07 ; 0,125^{*} \mathrm{p}^{2}=0,02$

Substituting these values in the formula, we obtain the inductance value:

$$
\begin{aligned}
& \text { At } \mathrm{n}=4 \\
& \mathrm{~L}=\frac{2}{n} \mu_{0} \omega^{2} \mathrm{c}\left(\operatorname{Ln} \frac{1}{p}+0,726+0,1776 \mathrm{p}+0,125 \mathrm{p}^{2}\right)
\end{aligned}
$$

$$
\mathrm{L}=\frac{2}{\pi} \times 4 \pi \times 10^{-7} \times 25 \quad \times 0,085
$$$$
(0,9+0,726+0,07+0,02)
$$$$
\mathrm{L}=3 \mu \mathrm{H}
$$$$
\mathrm{Sm}=33 \mathrm{~cm}^{2}-\text { coil conductor area }
$$

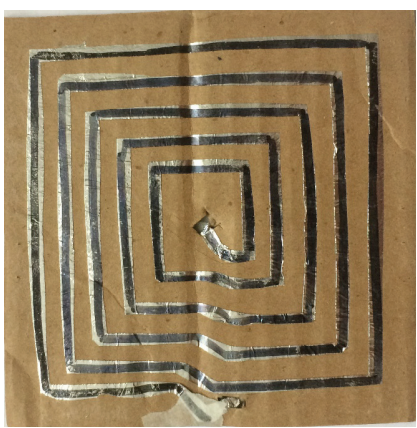

Fig. 16. Square shape flat coil

2) Square shape flat coil:

$\mathrm{c}=13 \mathrm{~cm} ; \mathrm{r}=5 \mathrm{~cm} ; \omega=6 ; \mathrm{D}=\frac{13}{\sin \frac{\pi}{n}}=18 \mathrm{~cm}$;

$\mathrm{r} / \mathrm{D}=\frac{5}{18}=0,3 ; \mathrm{d}=0,8075 \times 18=15 \mathrm{~cm} ; \mathrm{p}=\frac{5}{13,5}=0,4$;

$\operatorname{Ln} \frac{1}{0,4}=0,9 ; 0,1776^{*} \mathrm{p}=0,07 ; 0,125^{*} \mathrm{p}^{2}=0,02 ;$

$\mathrm{L}=\frac{2}{\pi} \times \mu_{0} \times 36 \times 0,13 \times 1,716=6,7 \mu \mathrm{H}$;

$\mathrm{Sm}=80 \mathrm{~cm}^{2}$

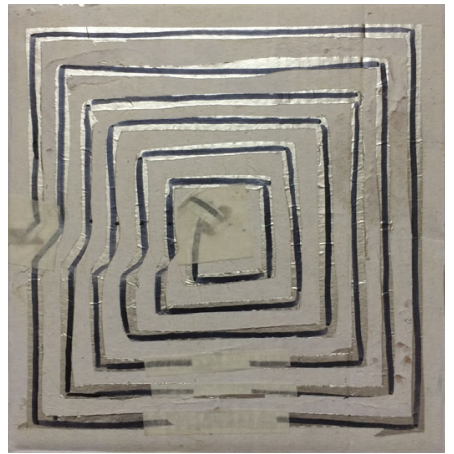

Fig. 17. Square shape flat coil

3) Rectangular s pe flat coil

$\mathrm{c}=14 \mathrm{~cm} ; \mathrm{r}=4,5 \mathrm{~cm} ; \omega=7 ; \mathrm{D}=\frac{14}{\sin \frac{\pi}{0,4}}=20 \mathrm{~cm} ;$

$\mathrm{r} / \mathrm{D}=\frac{4,5}{20}=0,3 ; \mathrm{d}=0,8075 \times 20=16 \mathrm{~cm} ; \mathrm{p}=\frac{4,5}{14}=0,3$;

$\operatorname{Ln} \frac{1}{0,3}=1,2 ; 0,1776^{*} \mathrm{p}=0,05 ; 0,125^{*} \mathrm{p}^{2}=0,01 ;$

$\mathrm{L}=\frac{2}{\pi} \times 4 \pi \times 10^{-7} \times 49 \times 0,14 \times 1,986=9,4 \mu \mathrm{H}$

$\mathrm{Sm}=94 \mathrm{~cm}^{2}$ 


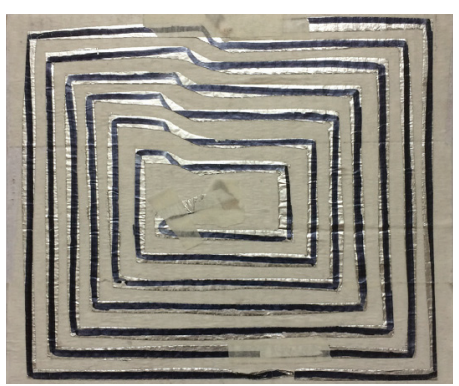

Fig. 18. The rectangular shape of a flat coil

4) Hexagonal shape flat coil:

$\mathrm{c}=7,5 \mathrm{~cm} ; \mathrm{r}=5 \mathrm{~cm} ; \omega=6 ; \mathrm{n}=6 ; \mu_{0=} 4 \pi \times 10^{-7} ;$

$\mathrm{D}=\frac{c}{\sin \frac{\pi}{n}} ; \mathrm{D}=\frac{7,5}{\sin \frac{3,14}{6}}=15 \mathrm{~cm} ; \mathrm{r} / \mathrm{D}=\frac{5}{15}=0,3 ;$

$\mathrm{d}=0,9124 \times 15=14 \mathrm{~cm} ; \mathrm{p}=\frac{r}{c}=\frac{5}{7,5}=0,6 ; \operatorname{Ln} \frac{1}{p}=0,51$;

$0,0678 * \mathrm{p}=0,04 ; 0,0491 * \mathrm{p}^{2}=0,017$;

$\mathrm{L}=\frac{3}{\pi} \mu_{0} \omega^{2} \mathrm{c}\left(\operatorname{Ln} \frac{1}{p}+1,3485+0,0678 \mathrm{p}+0,0451 \mathrm{p}^{2}\right)=$

$=\frac{3}{3,14} \times 4 \pi \times 10^{-7} \times 36 \times 0,075(0,51+1,349+0,04+$

$+0,017)=6,6 \mu \mathrm{H} ; \mathrm{Sm}=80 \mathrm{~cm}^{2}$

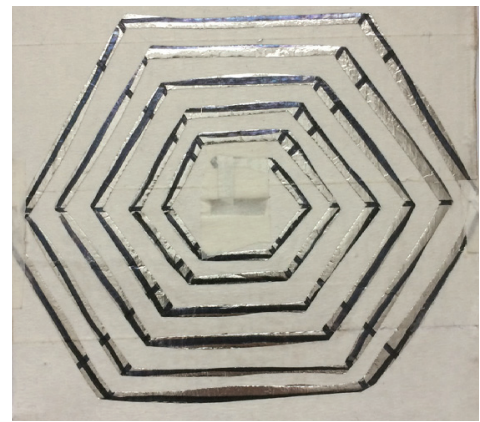

Fig. 19. Hexagonal shape of a flat coil

5) Octagonal flat coil shape:

$\mathrm{c}=6 \mathrm{~cm} ; \mathrm{r}=5 \mathrm{~cm} ; \omega=6 ; \mathrm{n}=8 ; \mathrm{D}=\frac{6}{\sin \frac{3,14}{6}}=16 \mathrm{~cm}$;

$\mathrm{r} / \mathrm{D}=\frac{5}{16}=0,3 ; \mathrm{d}=0,9501 \times 16=15 \mathrm{~cm} ; \mathrm{p}=\frac{r}{c}=\frac{5}{6}=0,8$;

$\operatorname{Ln} \frac{1}{p}=0,2 ; 0,0363 * \mathrm{p}=0,029 ; 0,0277 * \mathrm{p}^{2}=0,017 ;$

$\mathrm{L}=\frac{4}{n} \mu_{0} \omega^{2} \mathrm{c}\left(\operatorname{Ln} \frac{1}{8}+1,712+0,0363 \mathrm{p}+0,0277 \mathrm{p}^{2}\right)=$

$=\frac{4}{3,14} \times 4 \pi \times 10^{-7} \times 36 \times 0,06 \times(0,2+1,712+0,029+$

$+0,017)=7 \mu \mathrm{H} ; \mathrm{Sm}=86 \mathrm{~cm}^{2}$

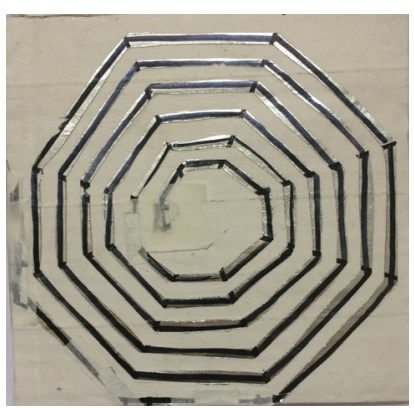

Fig. 20. Octagonal flat reel shape

6) Spiral shape flat coil:

$\mathrm{d}_{1}=13 \mathrm{~cm} ; \mathrm{d}_{2}=3 \mathrm{~cm} ; \mathrm{r}=5 \mathrm{~cm} ; \omega=6 ; \mathrm{d}=\frac{\mathrm{d} 1+\mathrm{d} 2}{2}=8 \mathrm{~cm}$; $\mathrm{p}=\frac{r}{d}=\frac{5}{8}=0,6 ; \pi=18,814 ; \pi=\frac{3}{13}=0,25$;

$\pi=\frac{(1+0,6)^{3}}{0,6^{2}}\left(1,7424+3,29 \times 0,25^{3} \operatorname{Ln} 0,2-2,27 \times(0,2)^{3}=19 ;\right.$ $\mathrm{L}=\frac{4 \pi \times 10^{-7}}{8 \pi} \times 36 \times 0,08 \times 19=2,7 \mu \mathrm{H} ; \mathrm{Sm}=73 \mathrm{~cm}^{2}$

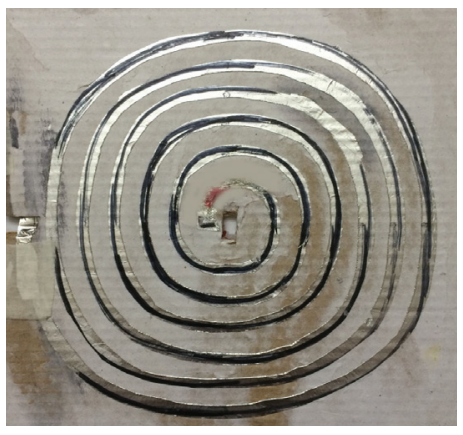

Fig. 21. Spiral shape flat coil

7) Spiral shape flat coil:

$\mathrm{d}_{1}=15 \mathrm{~cm} ; \mathrm{d}_{2}=3 \mathrm{~cm} ; \mathrm{r}=6 \mathrm{~cm} ; \omega=6 ; \mathrm{d}=\frac{18}{2}=9 \mathrm{~cm}$;

$\mathrm{p}=\frac{r}{d}=\frac{6}{9}=0,7 ; \pi=18,814 ; \pi=\frac{1-p}{11 p}+\frac{1-0,7}{1+0,7}=0,2$;

$\pi=17,032 ; \pi=\frac{(1+0,7)^{3}}{0,7^{2}}(1,7424+3,2900 \times$

$\times 0,2^{2} \operatorname{Ln} 0,2-2,27 \times(0,2)^{3}=17 ; \mathrm{L}=\frac{4 \pi \times 10^{-7}}{8 \pi} \times 36 \times$ $\times 0,09 \times 17=2,8 \mu \mathrm{H} ; \mathrm{Sm}=110 \mathrm{~cm}^{2}$

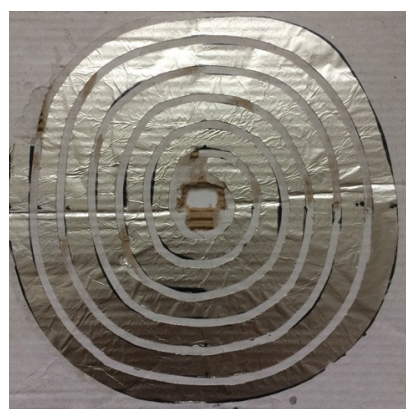

Fig. 22. Spiral shape flat coil 
8) Spiral shape flat coil:

$\mathrm{d}_{1}=10 \mathrm{~cm} ; \mathrm{d}_{2}=1,5 \mathrm{~cm} ; \mathrm{r}=4 \mathrm{~cm} ; \omega=6 ; \mathrm{d}=\frac{11,5}{2}=6 \mathrm{~cm}$; $\mathrm{p}=\frac{r}{d}=\frac{4}{6}=0,6 ; \pi=18,814 ; \pi=\frac{1-p}{1+p}+\frac{1-0,6}{1+0,6}=0,25 ;$

$\pi=18,614 ; \pi=\frac{(1+0,6)^{3}}{0,6^{2}}(1,7424+3,2900 \times$

$\times 0,25^{2} \operatorname{Ln} 0,25-2,27 \times(0,25)^{3}=19 ; \mathrm{L}=\frac{4 \pi \times 10^{-7}}{8 \pi} \times 36 \times$ $\times 0,06 \times 19=2,1 \mu \mathrm{H} ; \mathrm{Sm}=41 \mathrm{~cm}^{2}$

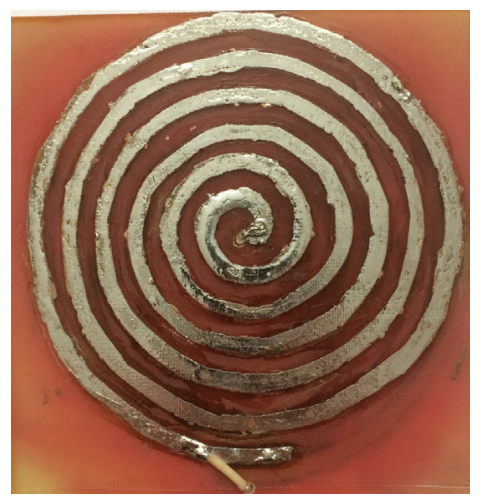

Fig. 23. Spiral shape flat coil

9) Spiral shape flat coil:

$\mathrm{d}_{1}=13 \mathrm{~cm} ; \mathrm{d}_{2}=3 \mathrm{~cm} ; \mathrm{r}=5 \mathrm{~cm} ; \omega=6 ; \mathrm{d}=\frac{16}{2}=8 \mathrm{~cm}$;

$\mathrm{p}=\frac{r}{d}=\frac{b}{8}=0,6 ; \pi=19 ; \pi=\frac{3}{13}=0,25 ; \pi=19$;

$\mathrm{L}=\frac{4 \pi \times 10^{-7}}{8 \pi} \times 36 \times 0,08 \times 19=2,7 \mu \mathrm{H} ; \mathrm{Sm}=73 \mathrm{~cm}^{2}$

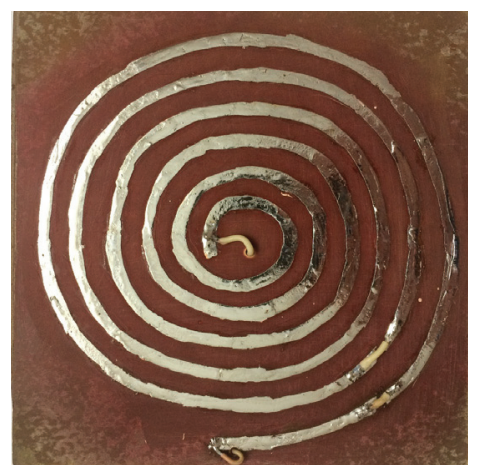

Fig. 24. Spiral flat coil shape

10) $\mathrm{S}$ iral shape flat coil:

$\mathrm{d}_{1}=12 \mathrm{~cm} ; \mathrm{d}_{2}=6 \mathrm{~cm} ; \mathrm{r}=3 \mathrm{~cm} ; \omega=7 ; \mathrm{d}=\frac{18}{2}=9 \mathrm{~cm} ;$

$\mathrm{p}=\frac{r}{d}={ }_{9}^{3}=0,3 ; \quad=26,56 ; \mathrm{L}=\frac{4 \pi \times 10^{-7}}{8 \pi} \times 49 \times 0,09 \times$

$\times 26,56=5,8 \mu \mathrm{H} ; \mathrm{Sm}=36 \mathrm{~cm}^{2}$

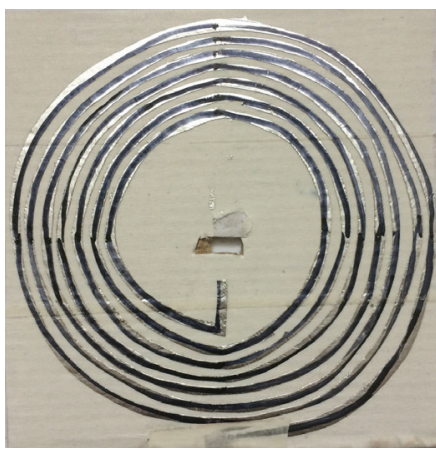

Fig. 25. Spiral shape flat coil

Table 2

Flat coil parameters

\begin{tabular}{|c|c|c|c|c|c|}
\hline & $\mathbf{d}_{\mathbf{1}}, \mathbf{c m}$ & $\mathbf{d}_{\mathbf{2}}, \mathbf{c m}$ & $\mathbf{r}, \mathbf{c m}$ & $\mathbf{\omega}$ & $\mathbf{L}, \boldsymbol{\mu H}$ \\
\hline $\mathbf{1}$ & 9 & 2,5 & 3 & 5 & 3 \\
\hline $\mathbf{2}$ & 13 & & 5 & 6 & 6,4 \\
\hline $\mathbf{3}$ & 14 & & 4,5 & 7 & 9,4 \\
\hline $\mathbf{4}$ & 12 & & 4,5 & 6 & 6,6 \\
\hline $\mathbf{5}$ & 14 & & 5 & 7 & 7 \\
\hline $\mathbf{6}$ & 12,5 & 3 & 5 & 6 & 2,7 \\
\hline $\mathbf{7}$ & 14 & & 5,5 & 6 & 2,8 \\
\hline $\mathbf{8}$ & 10 & 1 & 4 & 6 & 2,1 \\
\hline $\mathbf{9}$ & 13 & & 5 & 6 & 2,7 \\
\hline $\mathbf{1 0}$ & 12 & & 3 & 7 & 5,8 \\
\hline
\end{tabular}

\section{ANALYSIS OF DATA RECEIVED}

To study these structures, two coils were used, located at a distance of $20 \mathrm{~cm}$ from each other, were connected to a network analyzer to determine the resonant frequency of the structure of the modeling metamaterials, and a frequency from $7 \mathrm{MHz}$ to $30 \mathrm{MHz}$ was applied using a pulse generator. The wavelength of 10-43 m.

The shape of the structure modeling the metamaterials was chosen from numerous sets of geometric shapes (square, 6.8-angled, rectangular, and also spiral).

Table 3

Structure transfer factor at their location closer to the receiver

\begin{tabular}{|c|c|c|c|c|c|c|c|c|c|c|c|}
\hline \multicolumn{2}{|c|}{ № } & 1 & 2 & 3 & 4 & 5 & 6 & 7 & 8 & 9 & 10 \\
\hline \multicolumn{2}{|c|}{$\mathbf{L}, \boldsymbol{\mu H}$} & 6 & 3,4 & & 12,4 & 14 & 5,4 & 5,6 & 4,2 & 5,4 & 1 \\
\hline \multicolumn{2}{|c|}{$\mathrm{Sm}, \mathrm{cm}^{2}$} & 33 & & 94 & & 86 & & 110 & & 73 & 36 \\
\hline \multicolumn{2}{|c|}{$\mathrm{R}, \mathrm{cm}$} & \multicolumn{10}{|c|}{15} \\
\hline \multirow{9}{*}{ 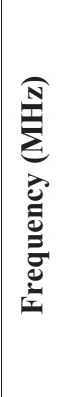 } & 7 & 1,1 & & 1,3 & & 1,2 & 1,4 & & & 1,4 & 1,7 \\
\hline & 10 & 1,1 & & & & 1,1 & & & & & 7 \\
\hline & 11 & 1,4 & 13 & 1,4 & 1,8 & 1,4 & 1,6 & 5 & & 0,3 & 1,4 \\
\hline & 12 & 1,1 & 1,2 & 1,2 & 2,5 & 1,5 & 1,7 & 2,9 & 0,4 & 0,5 & 0,4 \\
\hline & 14 & 1,2 & 1,4 & 1,4 & 1,8 & 2,3 & 4,4 & 0,2 & 0,6 & 0,4 & 0,3 \\
\hline & 16 & 1,1 & 1,2 & 1,2 & 1,3 & 1,5 & 3 & 0 & 1 & 0,8 & 0,9 \\
\hline & 18 & 1 & 1,5 & 1,8 & 0,3 & 4,8 & 0,8 & 0,4 & 0,7 & 0,5 & 0,6 \\
\hline & 20 & 1 & 1 & 2,2 & 2 & 0,7 & 0,5 & 0,5 & 0,7 & 0,7 & 0,7 \\
\hline & 30 & 0,8 & 0,8 & 0,8 & 0,8 & 0,5 & 0,8 & 0,8 & 0,8 & 0,8 & 0,8 \\
\hline
\end{tabular}




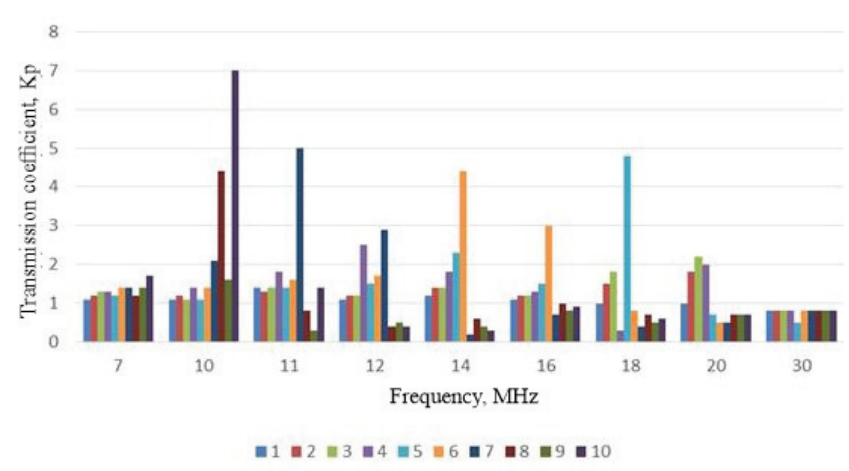

Fig. 26. The dependence of the transmission coefficient on the frequency (closer to the receiver)

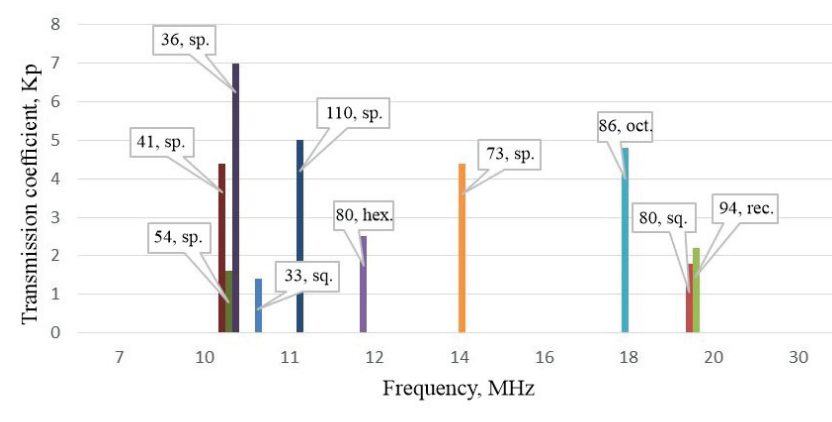

Fig. 27. Dependence of the transmission coefficient on the frequency (closer to the receiver)

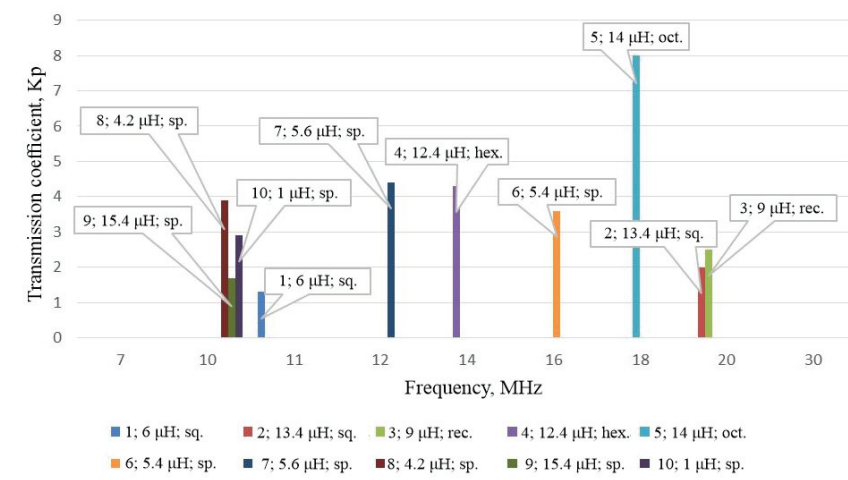

Fig. 28. The dependence of the transmission coefficient on the frequency (closer to the receiver)

Table 3

Structure transfer factor at their location closer to the receiver

\begin{tabular}{|c|c|c|c|c|c|c|c|c|c|c|c|}
\hline \multicolumn{2}{|c|}{ № } & 1 & 2 & 3 & 4 & 5 & 6 & 7 & 8 & 9 & 10 \\
\hline \multicolumn{2}{|c|}{$\mathbf{L}, \boldsymbol{\mu H}$} & 6 & 13,4 & 9 & 12,4 & 14 & 5,4 & 5,6 & 4,2 & 5,4 & 1 \\
\hline \multicolumn{2}{|c|}{$\mathrm{Sm}, \mathrm{cm}^{2}$} & 33 & & 94 & & 86 & 73 & 110 & 41 & & 36 \\
\hline \multicolumn{2}{|c|}{$\mathrm{R}, \mathrm{cm}$} & \multicolumn{10}{|c|}{15} \\
\hline \multirow{9}{*}{ 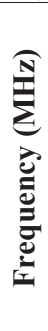 } & $\pi$ & 1,1 & 1,2 & 1,3 & 1,4 & 1,3 & 1,6 & 1,3 & 1,3 & & 1,3 \\
\hline & 1 & & & & & & & & & & 3 \\
\hline & 11 & & 1,3 & 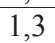 & & 3 & 1,4 & & & & 1 \\
\hline & 12 & 1 , & 1 & 1,2 & 2 & 1,3 & 1,5 & 2,7 & .5 & 5 & 0,5 \\
\hline & 14 & 1,2 & 12 & 1,3 & 3 & 1,6 & 2,7 & 0,2 & 0,8 & 6 & 0,6 \\
\hline & 16 & 1,1 & 1,2 & 1,2 & & 1,9 & 2,6 & 0,6 & 0 , & & 0,8 \\
\hline & 18 & 1 & 1,6 & 1,9 & 5 , & 6,4 & 0,4 & 0,4 & 0,7 & & 0,6 \\
\hline & 20 & 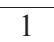 & 1,9 & 2,3 & 0 , & 0,4 & 0,5 & 0,6 & 0 , & 0,7 & 0,7 \\
\hline & 30 & 0,8 & 0,8 & 0,8 & 0,8 & 0,5 & 0,8 & 0,8 & 0,8 & 0,8 & 0,8 \\
\hline
\end{tabular}

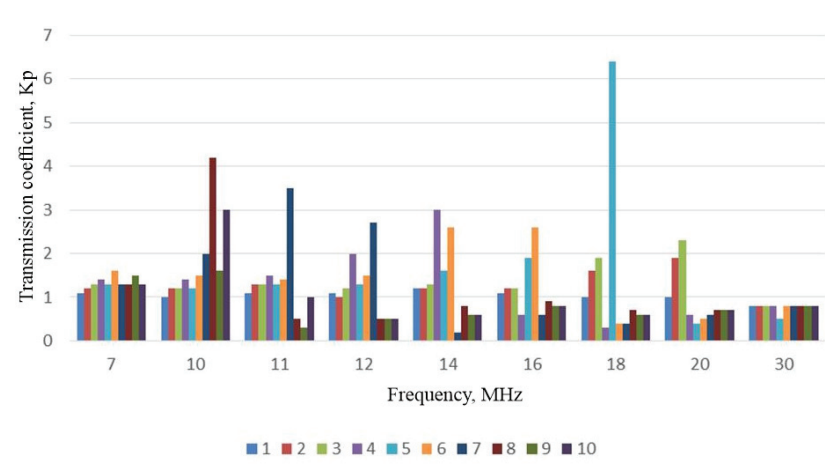

Fig. 29. The dependence of the transmission coefficient on the frequency (in the middle)

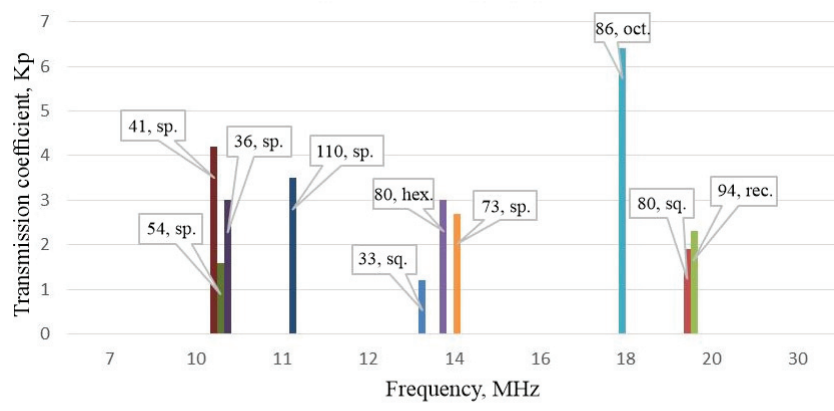

Fig. 30. The dependence of the transmission coefficient on the frequency (in the middle)

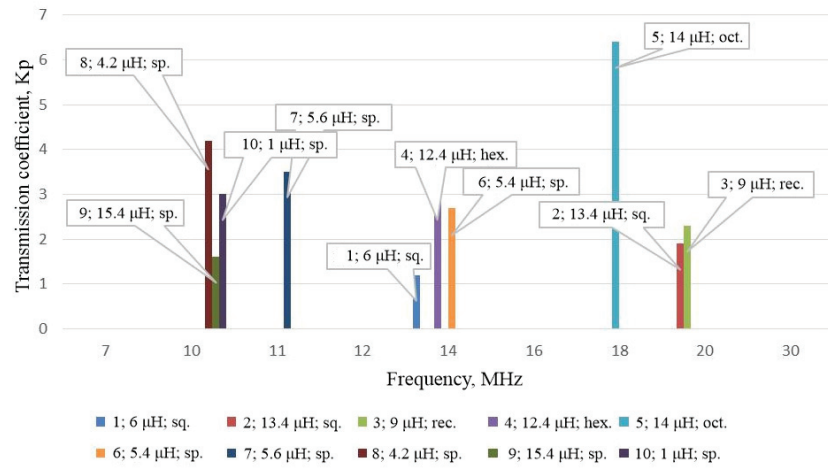

Fig. 31. The graph of the dependence of the transmission coefficient on the frequency (in the middle)

Based on the graph, we can conclude that when the structures are located in the middle between two coils, the coefficient decreases.

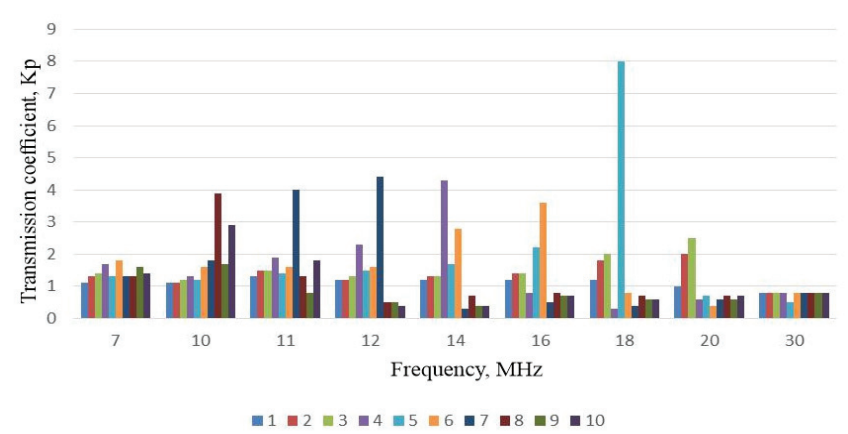

Fig. 32. The dependence of the transmission coefficient on the frequency (closer to the transmitter) 
Table 4

Structural transmission factor at their location closer to the transmitter

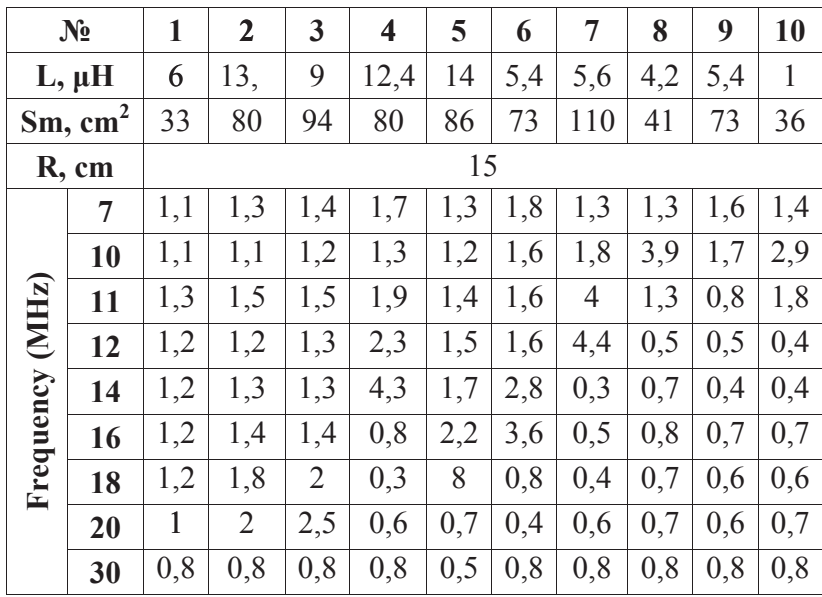

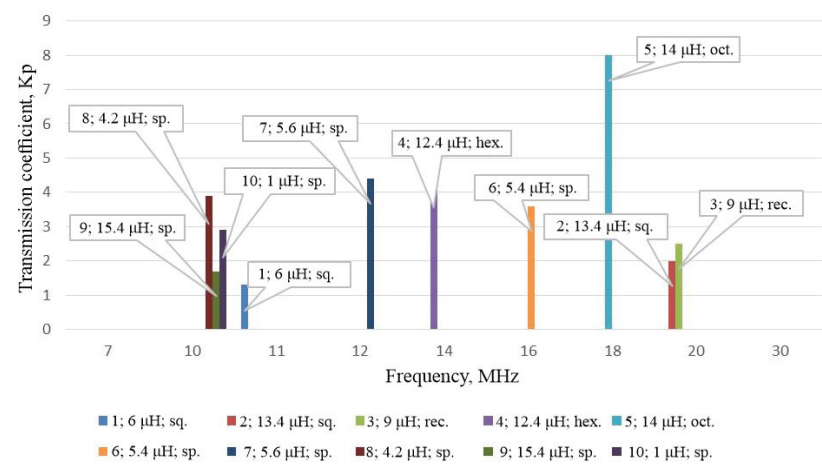

Fig. 33. The dependence of the transmission coefficient on the frequency (closer to the transmitter)

Based on the graph, we can conclude that each structure has its own resonant frequency. At a frequency of $18 \mathrm{MHz}$, the octagonal structure at No. 5 has the highest transmission coefficient $(\mathrm{Kp}=8)$ with an inductance of $14 \mu \mathrm{H}$ when located closer to the receiving coil. The structure No. 1 of a square shape has the smallest transmission coefficient $(K \mathrm{p}=1.3)$, since it has the smallest diameter and width of the conductor, unlike other structures. And for the rest of the structures modeling the metamaterial, the transmission coefficient is in the range from 1.5 to 4.5 .

Table 5

Structure transfer coefficient depending on their distance

\begin{tabular}{|c|c|c|c|c|}
\hline Frequency & № & $\begin{array}{c}\text { Number of } \\
\text { structures } \\
\text { m.m. }\end{array}$ & $\begin{array}{l}\text { Distance } R \\
\quad(\mathrm{~cm})\end{array}$ & $\begin{array}{c}\text { Gear ratio, } \\
\mathbf{K p}\end{array}$ \\
\hline \multirow{5}{*}{11} & & \multirow{5}{*}{ 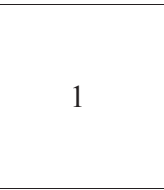 } & 10 & 5 \\
\hline & & & 15 & 2 \\
\hline & & & 20 & 1 \\
\hline & & & 25 & 1 \\
\hline & & & 30 & \\
\hline \multirow{5}{*}{11} & & \multirow{5}{*}{2} & 10 & \\
\hline & & & 15 & 11 \\
\hline & & & 20 & 6 \\
\hline & & & 25 & 3 \\
\hline & & & 30 & 2 \\
\hline
\end{tabular}

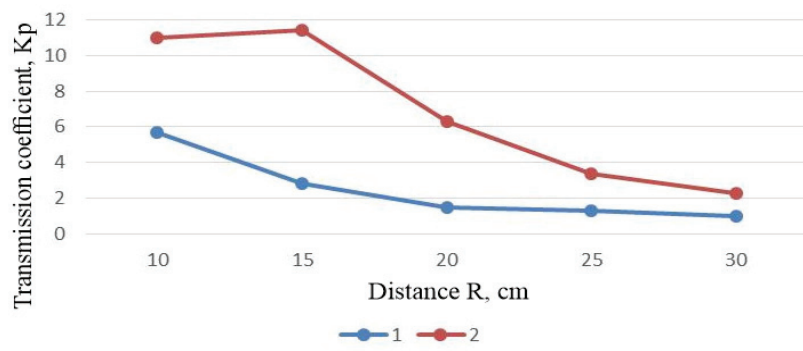

Fig. 34. The dependence of the gear ratio from $R$

On this graph, you can see that $\mathrm{Kp}$ increases by 2 times, 1 line (blue) characterizes Kp values without a structure, 2 line (red) with a structure modeling a metamaterial.

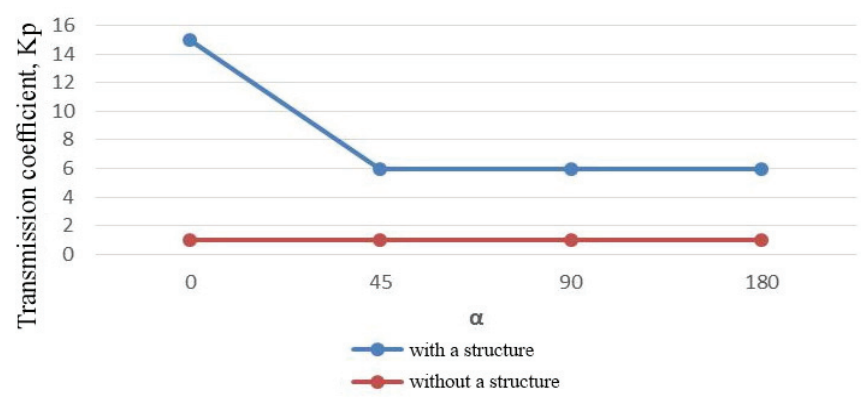

Fig. 35. The dependence of the gear ratio from the angle

Based on the graph, we can conclude that when the structures are rotated by a certain angle, the transmission coefficient slightly decreases, but not lower than the value of $K p=1$, but equal to 6 .

Table 6

Structure transfer coefficient depending on their angle

\begin{tabular}{|c|c|c|c|c|c|c|}
\hline \multirow{2}{*}{ Frequency } & \multirow{2}{*}{ № } & $\begin{array}{c}\text { Number of } \\
\text { structures } \\
\text { m. m. }\end{array}$ & \multirow{2}{*}{$\begin{array}{c}\text { Distance } \\
\mathbf{R} \text { (cm) }\end{array}$} & $\boldsymbol{\alpha}$ & \multicolumn{2}{|c|}{ Gear ratio, Kp } \\
\cline { 4 - 6 } & \multirow{2}{*}{11} & \multirow{2}{*}{20} & 0 & 1 & 15 \\
\cline { 4 - 6 } & & & 45 & & 6 \\
\cline { 4 - 6 } & & & 90 & & 6 \\
\cline { 4 - 6 } & & & 180 & & 6 \\
\hline
\end{tabular}

The following are tables comparing structures modeling a metamaterial with various parameters.

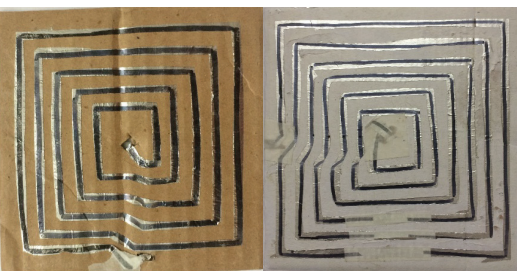

Ext. diameter $\mathrm{d}_{1}, \mathrm{~cm}$

Int. diameter $\mathrm{d}_{2}, \mathrm{~cm}$

Conductor width $\mathbf{r}, \mathrm{cm}$

Number of turns, $\omega$

Inductance $\mathrm{L}, \boldsymbol{\mu H}$

Frequency, $\mathrm{MHz}$

Coef. transmission $\mathrm{Kp}$

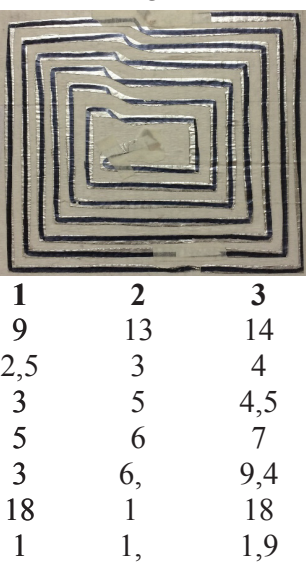

Fig. 36. Parameters of structures modeling the metamaterial No. $1,2,3$ 
4

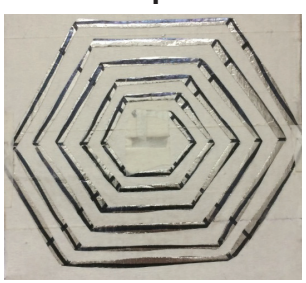

Ext. diameter $d_{1}, c m$

Int. diameter $\mathbf{d}_{2}, \mathbf{c m}$

Conductor width $\mathbf{r}, \mathrm{cm}$

Number of turns, $\omega$

Inductance $\mathbf{L}, \boldsymbol{\mu H}$

Frequency, $\mathrm{MHz}$

Coef. transmission Kp
5

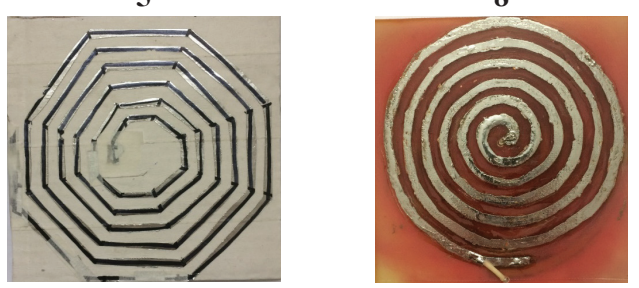

4

12

3

4,5

6

6,6

18

5,3
5

6,8

6,4

Fig. 37. The parameters of the structures modeling the metamaterial, the hexagonal shape on the left (No. 4), and the octagonal on the left (No. 5)

5

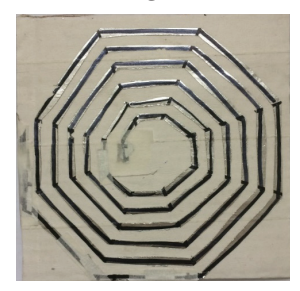

Ext. diameter $\mathrm{d}_{1}, \mathrm{~cm}$

Int. diameter $d_{2}, c m$

Conductor width $\mathbf{r}, \mathrm{cm}$

Number of turns, $\omega$

Inductance $\mathrm{L}, \boldsymbol{\mu H}$

Frequency, $\mathrm{MHz}$

Coef. transmission Kp

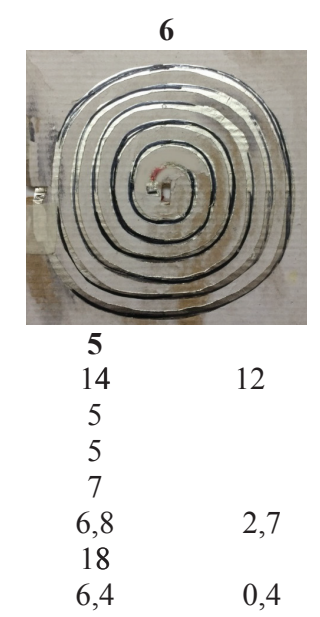

0,4

Fig. 38. The parameters of structures modeling the metamaterial, on the left is an octagonal shape (No. 5), and spiral on the left (No.6)

5

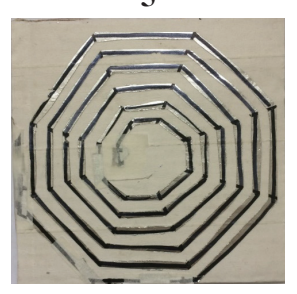

Ext. diameter $\mathrm{d}_{1}, \mathrm{~cm}$

Int. diameter $d_{2}, c m$

Conductor width $\mathbf{r}, \mathrm{cm}$

Number of turns, $\omega$

Inductance $\mathrm{L}, \boldsymbol{\mu H}$

Frequency, MHz

Coef. transmission Kp

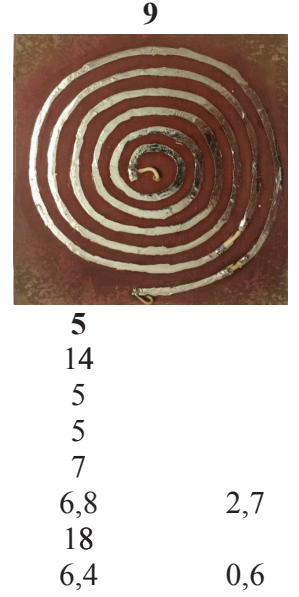

0,6

Fig. 39. The parameters of the structures modeling the metamaterial, the hexagonal shape on the left (No. 5) and the spiral on the left (No. 9)

Ext. diameter $d_{1}, \mathrm{~cm}$

Int. diameter $\mathrm{d}_{2}, \mathrm{~cm}$

Conductor width $\mathbf{r}, \mathrm{cm}$

Number of turns, $\omega$

Inductance $\mathrm{L}, \boldsymbol{\mu H}$

Frequency, $\mathrm{MHz}$

Coef. transmission Kp
10

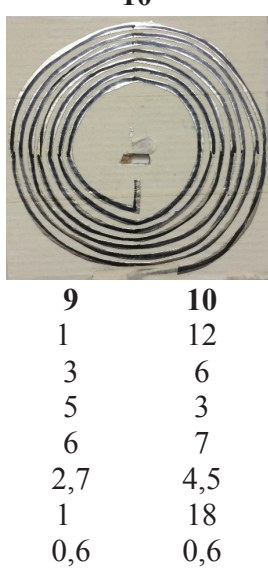

Fig. 40. Parameters of structures simulating a spiral-shaped metamaterial (No. 8, 9, 10)

Table 7

Parameters of structures modeling metamaterial

\begin{tabular}{|c|c|c|c|c|}
\hline \multirow[t]{2}{*}{$\mathbf{L}, \boldsymbol{\mu} \mathbf{H}$} & \multirow[t]{2}{*}{$\begin{array}{l}\mathrm{Sm} \\
\mathrm{cm}^{2}\end{array}$} & \multirow[t]{2}{*}{$\mathbf{R}, \mathbf{c m}$} & \multirow[t]{2}{*}{ № } & $\begin{array}{c}\text { Frequency } \\
(\mathrm{GHz})\end{array}$ \\
\hline & & & & 15 \\
\hline 6 & 33 & \multirow[t]{10}{*}{15} & 1 & -8 \\
\hline 13,4 & 80 & & 2 & -5 \\
\hline 9 & 9 & & 3 & -3 \\
\hline 12,4 & 80 & & 4 & -7 \\
\hline 14 & 8 & & 5 & -4 \\
\hline 5,4 & 73 & & 6 & -6 \\
\hline 5,6 & 110 & & 7 & -13 \\
\hline 4,2 & 41 & & 8 & -12 \\
\hline 5,4 & 54 & & 9 & -9 \\
\hline 1 & 3 & & 10 & -2 \\
\hline
\end{tabular}

This table shows the CR values of various structures at a frequency of $15 \mathrm{GHz}$. Based on the table, we can conclude that at this frequency the signal is attenuated, where the maximum attenuation value of structure 7 is $-13 \mathrm{~dB}$.

\section{CONCLUSION}

In the framework of this paper, we present the results of a study of the structures modeling metamaterials, as well as the features of the properties of the structure of metamaterials based on flat spiral coils.

The analysis of literary sources is carried out, the unique properties of metamaterials are studied, on the basis of which, modeling of structures modeling metamaterial is carried out.

The possibility of modeling the properties of metamaterials using structures from a cascade of flat coils placed in a magnetic field is considered. The possibility of such an approach proceeds from the assumption that for atoms of the crystal lattice charged particles moving in orbits form a magnetic field that interacts with the magnetic field of a neighboring atom.

Thus, the transfer of electromagnetic energy with frequencies having wavelengths is much greater than the step of the crystal lattice. This approach allows the use of flat coils as an element of the model of the structure of matter. 
By placing the coils in a certain sequence, one can achieve both the usual electromagnetic properties of the substance and the anomalous ones that we observe with metamaterials. It was found that short-circuited passive flat coils located in a certain order not only weaken the transmitted signal, but increase it under certain conditions, namely when they are closer to the transmitter and when the internal conductors are open.

Based on the experiments, it can be concluded that when the metamaterial is placed between these two coils, the transmission distance can be significantly increased by controlling and manipulating the near-field wave, which usually decays quickly from the source. This indicates that transmission efficiency and distance range can be improved by increasing the amount of structure of modeling metamaterials.
A study of the obtained structures revealed that resonant coupling can be realized when the transmitter and receiver are tuned to the same or similar resonant frequency.

\section{REFERENCES}

[1] J. Franklin. Double Negative Materials (DNM), Phenomena and Applications. Science. 2009.

[2] O. Baklickaya. Obratnyj effekt Doplera stal vidimym. Nauka i zhizn'. 2005-2019.

[3] S.N. Galyamin, A.V. Tyuhti. Obratnoe cherenkovsko - perekhodn e izluchenie zaryada, vletayushchego v anizotropnuyu sredu. Pis'ma v "ZHurnal tekhnicheskoj fiziki". 2011. Vol. 37 (7). P. 54-63.

[4] M.Yu. Zvezdina. Primenenie innovacij pri razrabotke radiotekhnicheskih sistem. Kollektivnaya monografiya. Izd. dom Akademii Estestvoznaniya, 2015. 224 p. 